\title{
EDITORIAL
}

\section{SOBRE EL MAESTRO, EL ENTRENAMIENTO, EL CUIDADO DE SÍ Y EL DECIR VERAZ}

Estas notas editoriales hacen un reconocimiento al maestro como forma y sujeto de la educación contemporánea que sobrevive a los embates de la mercantilización y el mercado que circula en la sociedad —en términos generales - y en la educación — como caso particular-

El filósofo alemán Peter Sloterdijk (2012), en Has de cambiar tu vida, explora la categoría antropotécnica, de manera novedosa, que se articula con las preocupaciones y posibilidades de la educación. Antropotécnica se refiere a "los procedimientos de ejercitación físicos y mentales con los que los hombres de las culturas más dispares han intentado optimizar su estado inmunológico frente a los vagos riesgos de la vida y agudezas de la muerte" (p. 24). El autor profundiza su postura y muestra cómo la antropotécnica se relaciona con la posibilidad del hombre de construir formas inmunes: armaduras, cascarones, formas simbólicas de defensa de sus entornos. Es el hombre ético, repetitivo, artista, incorporado en el training - o entrenamiento continuo-, en el ejercicio, lo que, desde el punto de vista antropológico, hace que el trabajo-ejercicio sea del hombre consigo mismo, es decir, en un esfuerzo de trazar puentes entre naturaleza y cultura. El ejercicio comporta no solamente acciones, sino también formas de relación consigo mismo y con los otros. Habilita al hombre como sujeto capaz de hacer cosas. En este sentido, la cultura y la educación asumen formas propias de adiestramiento, cuyo fin es la transmisión de contenidos intelectuales y éticos a las generaciones siguientes, de modo que puedan asumirlos para integrarse con mayor éxito en el contexto concreto al que pertenecen. Esta transmisión 
posibilita la idea y práctica de cultivo, que hace referencia al cuidado de reproducir la semejanza en los herederos. Sin este cuidado las posibilidades de la cultura toman otros rumbos. Aquí aparece un aspecto clave: la constitución de modelos transmitidos en el imaginario colectivo de los pueblos — héroes, monjes, deportistas, artistas...que marcan el derrotero hacia donde las nuevas generaciones deben mirar como forma de conservar e innovar dentro de la conservación. Este ejercicio de admiración produce que la educación inicialmente estuviese más cercana a la seducción, al maravillarse o sumergirse en los mundos posibles de la consolidación de las culturas. Aquí aparece la figura del maestro o entrenador, como lo llama Sloterdijk, que ha transitado por varias culturas con la función de orientar e incitar a los nuevos a través de metas, propósitos, modelos a lograr lo improbable, es decir, descifrar y posibilitar la transmisión de valores, formas y prácticas que aparentemente son ideales y que a través de la acción del maestro pueden ser realizables.

En este ámbito de la cultura aparece el entrenador como un guía de lo improbable, de lo no alcanzable, dentro de la paradoja entre fines inconmensurables e investigación para ingresar en ese camino. "Desde el punto de vista sistémico, le compete la tarea de hacer visible la paradoja de la cultura de que sea justamente imposible de imitar lo que se emplea como acicate para la imitación más intensiva" (Sloterdijk, 2012, p. 352). El entrenador - maestro- aparece en esta escena de la cultura como ese ser excepcional que logró, a través del ejercicio, el milagro de la autotransformación. Es excepcional, diferente, inalcanzable, pero, además, seduce e invita. Congrega y forma discípulos, mas con ciertos límites: selecciona, jerarquiza, escoge. Clasificar separa los aptos de los no aptos, los más dotados de los menos dotados, los más competentes de los menos competentes. Sloterdijk reconoce diez tipos de trainers - entrenadores o maestrosque no solo se insertan en la cultura para su transmisión, sino, a la vez, reproducen el principio de convertir su tarea en algo realizable e imitable, así estén convencidos de la improbabilidad de realización y de su concreción en la realidad.

El primer caso es el gurú de la tradición brahmánico-hinduista, el cual implica "un modo de enseñar y aprender basado en la iniciación y 
la extralimitación hacia una esfera del saber sagrado y no-público" (Sloterdijk, 2012, p. 354). El segundo caso es el maestro budista: aquí no está tanto en juego la transmisión de una doctrina religiosa, sino la superación de las condiciones del sufrimiento, para llegar al punto culminante, su liberación, es decir, desplazar el sacrificio y el sufrimiento al ámbito interior. Luego aparece el apóstol que se subsume en la imitación de Cristo para que, al lograr esta condición, otros lo imiten: funge en la comunidad como un prototipo y alguien que puede imprimir su sello. Debe tener una vida ejemplar y ejemplarizante. Otro tipo de maestro que aparece es el filósofo, que se fragmenta en tres: el erótico, representado en la figura de Sócrates, que seduce al servicio del ideal que es la propia sabiduría; el estatuario, representado por marco Aurelio como el filósofo práctico, en la acepción antigua, el sabio ascético. Ocupa un lugar central el entrenamiento entre el cuidado filosófico de sí mismo y la constitución de su interior, como el escultor que cincela delicadamente la estatua interior. Por último, está el gnóstico representado en Plotino, el maestro de las ascensiones al logos por encima del mundo físico. La quinta figura de maestro es el sofista como experto universal que logra dar el salto entre lo lingüístico y lo vital, para lograr un saber total aplicado. Es un experto del conocimiento, sabe todo y "es capaz de todo lo perteneciente a una actitud vital superior" (Sloterdijk, 2012, p. 369). A estos cinco primeros tipos de entrenadores, nuestro filósofo los denomina espirituales.

El segundo grupo al cual se refiere es a los maestros pragmáticos, que tendrían que ver con la transmisión de técnicas especiales y de todo un complejo de saberes orientados a la praxis. El primero es el entrenador de atletas, que imprime en su voluntad de éxito su propia voluntad. Cuando la primera incrementa, le ayuda a superar los momentos difíciles. El segundo tipo de maestro pragmático es el artesano, reactualizado por Sennett (2009). El principio que le rige tiene en cuenta la obra del artesano en la coincidencia entre ejercicio y producción. En otras palabras, este es el maestro de la repetición, de la paciencia, del cuidado y de la vigilancia.

Tal actividad en el campo de la artificialidad, que se ha convertido en algo anónimo y degradado, proporcionaría los presupuestos ideales 
para el surgimiento de un tipo de producción asentado exactamente en los límites entre la fabricación y la meditación. Esto estimularía una labor del ejercitante donde el agente reproduce y amplía su propia competencia en el ejercicio de esa actividad a medida que se enfrasca en la fabricación del objeto o en la consecución del efecto buscado (Sloterdijk, 2012).

Sloterdijk concluye su recorrido haciendo referencia a tres tipos con licencia para enseñar en un campo pragmático: los profesores de las universidades, los maestros de la escuela primaria y los escritores, comprometidos en el "drama de la autosuperación progresiva del privilegio de la cultura o de la democratización de las elites" (Sloterdijk, 2012, p. 377). La preocupación de Sloterdijk es sobre el hecho de que muchas de estas élites se han prescrito a sí mismas con el designio de hacer superfluo el entrenamiento mediante el entrenamiento, es decir, la potencia del ejercicio es subsumida por el mismo ejercicio como algo sin sentido, sin valor agregado, sin creación: la repetición por la repetición o solo por el rendimiento y la acumulación.

\section{II}

Foucault, que no fue propiamente un estudioso de la pedagogía y la educación, plantea en la última parte de su trabajo - conocido como el último Foucault (Pulido \& Espinel, 2017) o el Foucault de la éticaalgunas reflexiones que pueden ayudar a comprender la actualidad del maestro y sus posibilidades de afectar la contemporaneidad. El filósofo, recordando a Sócrates, señala la importancia del maestro, cuya misión es interpelar, incitar a los otros a que cuiden de sí mismos, se ocupen de cada uno y puedan ejercer sobre sí formas de gobierno que puedan decidir su constitución como sujetos en las acciones concretas. El ocuparse de sí (González \& Pulido, 2014) en la perspectiva socrática tiene tres ejes de anclaje: el primero hace referencia a la responsabilidad del maestro en la incitación y recuerdo constante a los otros sobre su propio cuidado; el segundo se refiere a la posibilidades propias de su ejercicio, el maestro jerarquiza sus acciones, moviliza todo su ser al servicio de incitar al otro; el tercero consiste en lograr en el otro el despertar hacia su propio cuidado. Por eso surge la comparación de Sócrates con el tábano, aquel "insecto que persigue a los animales, 
los pica, los hace correr y agitarse" (Foucault, 2002, p. 24). De aquí que la preocupación, inquietud y cuidado de sí mismo es una espina, aguijón que debe ser clavada en la carne y el espíritu de los hombres para lograr movilidad, transformación, despertar; dolor que permita salir del letargo de la comodidad que las distracciones y las seducciones de las sociedades ubican en el camino del conocimiento y el saber. La inquietud de sí está mediada por lo que los griegos llamaban la actitud filosófica, una manera de vivir y de transitar. El maestro que logra la actitud filosófica hace posible que el saber no sea solamente un artificio en la formación y en la constitución como sujetos. El saber en esta perspectiva se convierte en la posibilidad de vivir. El maestro solo logra inquietar a los otros en la medida que su actitud sea filosófica en el sentido de vivir con el saber, para el saber y desde el saber.

La otra arista de la problemática del maestro planteada por Foucault es sobre el enseñar y la enseñanza misma: qué se enseña y cuáles son la posibilidades del maestro para hacerlo. Foucault acude a preguntar por el discurso del maestro y por el sitio de su enseñanza, que sería la verdad, no como esencia fundante de realidades y de homogeneización, sino la verdad sobre sí mismo. Acude a la noción griega de parrhesía, que alude desde su raíz etimológica a: franqueza, apertura de corazón, apertura de palabra, apertura de lenguaje, libertad de palabra.

El hablar franco es una actitud que determina la forma en la que el sujeto se relaciona consigo mismo y con los demás (González, 2017), actitud que representa coraje frente a la verdad, el cual significa a su vez el reconocimiento de un sujeto capaz de decir la verdad acerca de sí, reconocido como espiritual. Foucault indica que la parrbesía puede reconocerse como técnica, virtud y deber que sustenta dos tipos de sujetos: los que saben valerse de la parrbesía y los que no, sin olvidar que para el que se vale de ella es vital la presencia del otro. Esa verdad frente al otro presenta una serie de dualismos, en los cuales se refleja, por ejemplo en el marco de la dimensión pedagógica, la dualidad maestroestudiantes. Al referirse a la parrbesía como una dimensión pedagógica, se ubica al maestro como conductor de almas, el cual va a asumir el papel del parrhesiasta, que implica hacer uso del coraje para construirse como sujeto que dice la verdad sobre sí mismo, que a su vez moviliza en sus discípulos en ese ejercicio. El ejercicio del parrhesiasta requiere 
una armonía entre la verdad dicha y el pensamiento del sujeto que la expresa, generando un espacio de tensión entre los interlocutores, en la que el coraje se hace evidente, en tanto que el parrhesiasta, al exponer su verdad, pone en riesgo su relación con el otro.

De aquí que el maestro contemporáneo que pretenda serlo no solo debe ubicarse en el cuidado, la inquietud de sí como ejercicio de transformación del otro, sino que a la vez se ubica en el ejercicio del decir veraz que implica no solo un acompañamiento cercano, sino la posibilidad de ruptura frente a la tragedia de la verdad a las verdades que consolidan el ejercicio pedagógico y las prácticas educativas contemporáneas.

\section{III}

El fascículo 22 que los lectores tienen en sus manos corresponde al volumen 10 e inaugura el décimo año de actividades académicas de la revista Praxis \& Saber, un trayecto que ha sido posible gracias a la dedicación de muchas personas de los comités, asistentes, autores, evaluadores. En esta ocasión queremos hacer un homenaje muy especial a la directora y fundadora de la revista, la profesora Leonor Gómez Gomez. "Leíto", como afectuosamente la llamamos, en el año 2009, luego del 1er Congreso de Investigación y Pedagogía, se dio a la tarea de concretar las diferentes actividades de la Maestría en Educación de la UPTC — de la cual también es inspiradora y ejecutora- en productos que los participantes del programa pudiesen mostrar a la comunidad académica local, nacional e internacional. Por eso se da el sí a la publicación del primer número con algunos textos reunidos del congreso y otros artículos de profesores invitados. De la mano de Leíto nace este sueño académico de concretar en una publicación semestral investigaciones en el campo de la educación que sirvieran para su consolidación y para la visibilización de la UPTC, a través de la maestría y la nueva revista en el ámbito académico nacional. Este impulso de la profesora Leonor no se quedó solo en la publicación, sino que se logró subir otros escalones, como la clasificación en PUBLINDEX desde el año 2012 y presencia en índices comprensivos, bases de datos, repositorios. Estas cortas líneas quieren expresar el profundo agradecimiento a su desempeño y labor en la revista y la 
consolidación de la misma. 22 fascículos en 9 años son un gran logro y una empresa que sin la presencia de ella hubiese sido casi imposible.

¡Gracias, profesora Leonor!

\section{IV}

Los artículos del presente número están organizados en tres grandes categorías: inicialmente aparecen textos productos de investigaciones que abordan la formación como categoría de relación con las actividades de los maestros y sus desempeños en campos disciplinares diversos. Un segundo grupo de artículos aborda temas relacionados con el aprendizaje en el mundo contemporáneo. Por último, se muestra una interesante relación, en dos artículos, sobre las posibilidades educativas en agroindustria y agroecología.

En el primer conjunto de artículos está como eje de discusión la categoría formación y formación docente. Las profesoras Laura Galazzi, Diana Gómez y Muriel Vásquez de la Universidad de Buenos Aires ([UBA] presentan una investigación titulada Políticas mundializadas de formación docente: propuestas para una lectura filosófica, que pretende mostrar las políticas internacionales producidas en la OCDE y el Banco Mundial, que impactan las realidades formativas de maestros latinoamericanos. El análisis se hace en tres ejes: jerarquías socioeconómicas, supuestos de género y rasgos culturales moderados de futuros profesores. El segundo texto de este conjunto es el titulado La formación de los profesores de matemáticas un estudio de caso del profesor Alfonso Jiménez Espinosa de la UPTC, quien utiliza un enfoque cualitativo. Presenta las condiciones y posibilidades históricas de la formación de profesores de matemáticas en la UPTC. También utiliza como archivo planes de estudio, perfiles profesionales y documentos institucionales. De la Institución Educativa José Antonio Galán de Córdoba, las profesoras Edris Pizarro Gamero y Shirlena Gómez Muskus presentan un texto titulado Concepciones docentes sobre evaluación: de los lineamientos, el discurso y la práctica, que recoge los resultados de una investigación que involucra profesores de diferentes áreas del saber, de educación básica y media. Utilizó formas de investigación cualitativa para hacer un seguimiento sobre el impacto y las consecuencias de la implementación del Decreto 
1290 del Ministerio de Educación Nacional [MEN]. De la Universidad Católica de la Santísima Concepción de Chile, los profesores Claudine Glenda Benoit Ríos, Cristian Alonso Jaramillo y Ricardo Andrés Castro Cáceres comparten en la revista el texto titulado Aprendizaje y formación valórica en la enseñanza mediante tutorías entre pares que plantea como objetivo describir la percepción de tutores sobre la implementación de un programa de tutores-pares en una universidad de la Región del Biobío, Chile, y determinar las estrategias usadas para acompañar a estudiantes con bajo rendimiento académico. La metodología utilizada es de carácter cualitativo, los resultados de la investigación permiten visualizar la valoración que el tutor hace en torno a las habilidades y valores que se potencian en los participantes del programa.

La segunda parte del fascículo presenta un grupo de artículos que hacen énfasis en el aprendizaje, la educación para la paz y las posibilidades interdisciplinarias de la pedagogía en relación con la agroindustria y la agroecología. Las profesoras Angélica María Rodríguez Ortiz y Claudia Patricia Marín Ortiz, de la Universidad Autónoma de Manizales, comparten los resultados de una investigación titulada Implementación de un modelo de juego interactivo para aprender matemáticas, que a través de un juego-prototipo pretende desarrollar el pensamiento lógico-matemático, con una perspectiva cualitativa. Educación para la paz y formación profesional: una aproximación desde la investigación documental se titula el artículo que presenta los resultados de una investigación cualitativa de orden documental que enfatiza en la relación entre educación en y para la paz con la formación profesional en ciencias sociales. El trabajo es firmado por Myriam Fernanda Torres Gómez de la Universidad de la Salle. El profesor Juan Sebastián Angarita Zapata, de la Fundación Deusto de españa y Carlos A. Vásquez Cardozo y Hugo H. Andrade-Sosa de la Universidad Industrial de Santander, publica el artículo titulado Ampliando procesos y espacios de aprendizaje en agroindustria con dinámica de sistemas, que gira en torno al uso en clase de ambientes de aprendizaje, a través de una estrategia de integración del modelado y simulación con dinámica de sistemas [DS] a la formación en agroindustria, para complementar el aprendizaje de conocimiento teórico que permita acercamientos de los estudiantes a experiencias reales en campo. En la misma línea de trabajo, los profesores de la Universidad Nacional 
de Colombia, María José Pitta Paredes y Álvaro Acevedo Osorio, resaltan en el artículo titulado Contribuciones de la agroecología escolar a la soberania alimentaria: caso Fundación Viracocha (San Agustín - Colombia), a través de un estudio de caso, la importancia de la agroecología escolar para generar un cultura agroalimentaria en entornos escolares y comunitarios, con la relación de tres ejes de trabajo producción, transformación y consumo. La perspectiva metodológica del proyecto estuvo localizada en los enfoques cualitativos de investigación. Así mismo, las profesoras María de Fátima Ramos Andrade y Ana Silvia Moço Aparício de la Universidade Presbiteriana Mackenzie en Brasil, comparten un texto titulado Escrituras contemporáneas: el cine de animación, que hace una descripción de la escritura contemporánea a través del cine de animación, con la convicción de que historiar un determinado sistema de representación es una oportunidad de modelar, no solo algunos movimientos de la expansión de la cultura, sino también un ejercicio para entender como la cultura se transforma.

Para finalizar el fascículo, se presente el último grupo de artículos entre los cuales se encuentra el primer artículo titulado Los espacios de formación continua respecto a la enseñanza de lengua escrita, de Sandra Milena Díaz Chaves, de la Institución Educativa Rural Departamental Cacicazgo y Sandra Cecilia Arandia Rodríguez, de la Institución Educativa Departamental Integrado San Cayetano, el cual realizó un análisis de los antecedentes del ejercicio investigativo, mediante un proceso de indagación, que le sirvió para visibilizar los modelos de formación continua de los docentes, al igual que la transformación de prácticas en la enseñanza de la lengua escrita, identificando la importan de replantear espacios que tengan en cuenta los intereses de los estudiantes, como sus conocimientos previos y se trabaje los conocimientos partiendo de hipótesis. La investigadora Francia Terrazas Bañales, de la Universidad Autónoma de Chihuahua, México, en su artículo Motivación en estudiantes de música: orquesta de flautas de la facultad de artes presenta los resultados de las entrevistas realizadas de su proyecto denominado Orquesta de Flautas de la Facultad de Artes: Inclusión y trabajo grupal de estudiantes y docentes. Proyecto de investigación, en el cual se observa que la motivación intrínseca es predominante en un primer escenario y que a partir de esta los estudiantes desarrollaron sus habilidades, resolvieron problemas, disminuyeron el miedo escénico, y avanzaron en 
su aprendizaje académico. Por último, el artículo Percepción de la evaluación en estudiantes y docentes del programa de fisioterapia, de las investigadoras Zoraya Trinidad Cáceres Bermón, Olga Jeannette Munévar Ramírez y Luisa Liliana Lara Gómez, de la Universidad de Pamplona, expone las concepciones de la evaluación tanto de estudiantes como profesores del programa de Fisioterapia y su incidencia en las prácticas clínicas, donde se concluye la evaluación como un componente regulador que observa el desempeño del estudiante, pero a la vez reconoce sus avances, resalta sus aciertos y capacidades en su proceso formativo.

Invitamos a todos los lectores de Praxis \& Saber a leer, disfrutar, trabajar y citar para sus investigaciones este fascículo de Praxis \& Saber.

\title{
Referencias
}

Foucault, M. (2002). Hermenéutica del sujeto. México DC, México: Fondo de Cultura Económica.

González, B. (2017). La Noción de Parrhesía, el maestro y el cuidado de sí. Pulido, O \& Espinel, O (2017) Formas y expresiones metológicas en el último Foucault. Tunja, Colombia: Editorial UPTC

González, B., \& Pulido, O. (2014). Cuidado de sí como principio educativo. Educación y Ciencia, (17), 125-143.

Pulido, O., \& Espinel, O. (2017) Formas y expresiones metodológicas en el último Foucault. Tunja, Colombia: Editorial UPTC

Sennett, R. (2009). El artesano. Barcelona, España: Anagrama.

Sloterdijk, P. (2012). Has de cambiar tu vida: Sobre antropotécnica. Valencia, España: Pretextos. Oscar Pulido Cortés

\author{
Oscar Pulido Cortés \\ Doctor en Educación \\ Universidad Pedagógica y Tecnológica de Colombia \\ oscar.pulido@uptc.edu.co \\ https://orcid.org/0000-0002-3863-5007 \\ https://doi.org/10.19053/22160159.v10.n22.2019.9303
}

